\title{
Imaging of Synaptically Evoked Intrinsic Optical Signals in Hippocampal Slices
}

\author{
Brian A. MacVicar and Daryl Hochman \\ Neuroscience Research Group, University of Calgary, Calgary, Alberta, Canada T2N 4N1
}

Imaging analysis techniques were used to examine changes in the intrinsic optical properties in hippocampal brain slices that occurred during synaptic activity evoked by Schaffer collateral stimulation in CA1. Repetitive synaptic activity was associated with an increase in light transmission in the synaptic region in stratum radiatum. The effect was seen at wavelengths of light between 450 and $800 \mathrm{~nm}$ but was of greater amplitude at longer wavelengths. Blocking synaptic transmission with either $\mathrm{Ca}^{2+-f r e e ~ E G T A ~ p e r f u s a t e ~ o r ~ k y n-~}$ urenic acid (an excitatory amino acid antagonist) blocked the optical signal, indicating that it resulted from postsynaptic activation of the cells and was not due to presynaptic fiber volleys or transmitter release alone. Because the optical changes were blocked by reducing extracellular $\mathrm{Cl}^{-}$(by replacement with gluconate) or by furosemide (an anion transport inhibitor), increased $\mathrm{Cl}$ - transport (conceivably $\mathrm{Na}$ K-2Cl cotransport) may generate these signals possibly by causing cellular swelling and thereby less light scattering. These optical changes were not blocked, however, by bicarbonate-free solution, indicating that bicarbonate transport may not be involved. Changes in the intrinsic optical signal could be related to glial swelling due to $K+$ released during neuronal activity because high-K+-induced swelling of cultured astrocytes is blocked by furosemide and low-Clsolution. Intrinsic optical signals of neuronal tissue should be considered when voltage- or ion-sensitive dyes are used.

Changes in the intrinsic optical properties of nerve tissue have been reported to be correlated with neuronal activity (reviewed in Cohen, 1973; Grinvald et al., 1986; Lieke et al., 1989). Action potentials in nerves are associated with a transient increase in light scattering, followed by a long-lasting decrease (Hill and Keynes, 1949; Cohen and Keynes, 1971). Stimulation of appropriate sensory input to the somatosensory cortex of the rat or the visual cortex of the cat induces changes in reflectance in activated areas of the tissue (Grinvald et al., 1986). This has been used to image functional organization of the primate visual cortex (Ts'o et al., 1990). Neurosecretion from the mammalian neurohypophysis is correlated with changes in light-scattering properties (Salzberg et al., 1985; Obaid et al., 1989), and mem-

Received Aug. 28, 1990; revised Dec. 26, 1990; accepted Dec. 27, 1990.

This work was supported by the Medical Research Council (MRC) of Canada. B.A.M. is an Alberta Heritage Foundation for Medical Research Scholar and an MRC Scientist. We thank Dr. Q. Pittman and Dr. B. Ransom for comments on the manuscript.

Correspondence should be addressed to Brian A. MacVicar at the above address. Copyright (C) 1991 Society for Neuroscience 0270-6474/91/111458-12\$03.00/0 brane depolarization decreases reflectance in guinea pig cerebral cortical slices (Lipton, 1973). Photodiode measurement of light transmittance through hippocampal slices has indicated that synaptic transmission alters light scattering (Grinvald et al., 1982) and that spreading depression causes a decrease in light transmission (Snow et al., 1983). The cellular mechanisms underlying these changes in the intrinsic optical properties of neuronal tissue during activity are not precisely known as yet. The change in light scattering in axons is thought to represent axonal swelling during activity (Cohen and Keynes, 1971; Cohen, 1973). In the neurohypophysis, the change in light scattering is associated with neurosecretion because it is blocked by $\mathrm{Ca}^{2+}$ antagonists (Salzberg et al., 1985; Obaid et al., 1989). In the intact cortex, it has been suggested that one component of the intrinsic signal is associated with alterations in blood flow during activity (Lieke et al., 1989). Other components in the cortex are apparently independent of blood flow change and are possibly due to cellular swelling, ion movements, secretion, and changes in the extracellular space.

Cellular swelling would lead to decreased light scattering, decreased reflectance, and increased light transmittance through tissue (Cohen, 1973; Lipton, 1973). Shrinkage of the volume of the extracellular space (presumably due to cellular swelling) has been shown to occur during evoked activity in the cortex (Dietzel et al., 1980, 1982), in the hippocampal slice during synaptic stimulation (Heinemann et al., 1983) and during high-K ${ }^{+}$perfusion (McBain et al., 1990), in an experimental model of cpilepsy (Heinemann and Dietzel, 1984), and in the honeybee drone retina during light illumination (Orkand et al., 1984). Also in the isolated optic nerve, direct electrical stimulation induces swelling, which alters the volume of the extracellular space (Ransom et al., 1985). The onset of this phenomenon in the optic nerve during development occurs when astrocytes are present following postnatal proliferation, suggesting that astrocyte swelling is responsible for extracellular volume changes. In the CNS, high- $\mathrm{K}^{+}$-induced cellular swelling is confined mainly to astrocytes (reviewed in Hertz, 1981; Kimelberg and Ransom, 1986). Although neuronal swelling occurs under some circumstances such as during spreading depression (Van Harreveld and Khattab, 1967), dircct measurements of cell volume have shown activity-dependent shrinkage in motoneurons in frog spinal cords (Serve et al., 1988). Studies have generally indicated that astrocytes are the cellular element that is most sensitive to swelling. Therefore, a component of the stimulus-evoked changes in intrinsic optical properties of the CNS tissue could be due to activity-dependent astrocyte swelling.

The mechanisms of astrocyte swelling have been well studied in cell culture, and $\mathrm{Na}-\mathrm{K}-2 \mathrm{Cl}$ cotransport (Geck et al., 1980; Geck and Heinz, 1986) has been shown to play a major role in 


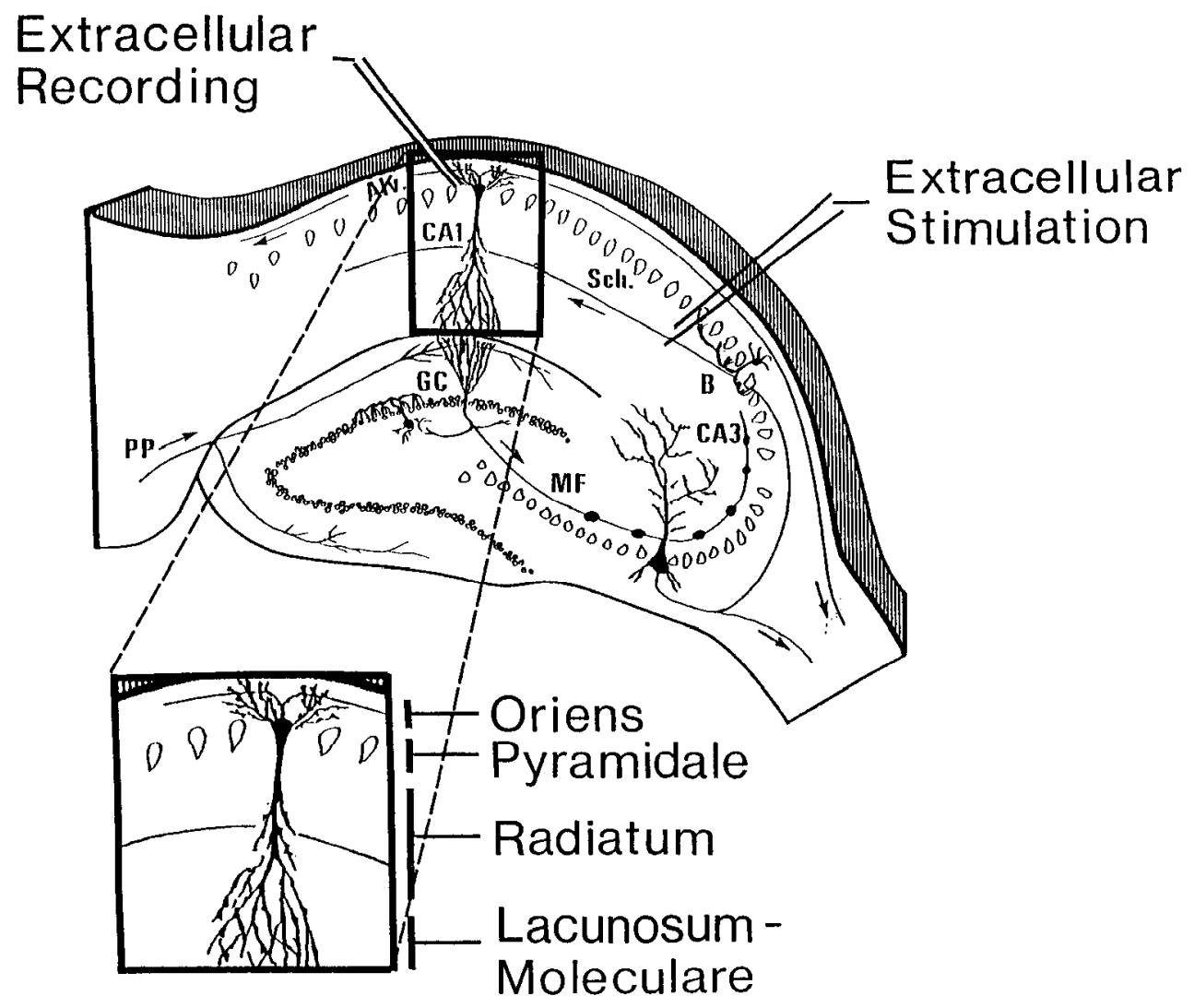

Figure 1. Schematic illustrating the area of recording and video imaging of hippocampal slices. Video images were obtained from the CAl region that is demarcated by a box and is shown in expanded scale in the inset below. The images always included the CA1 layers labeled in the inset, including the stratum oriens, pyramidale, radiatum, and lacunosum-moleculare. Sometimes the dendritic region of the granule cells in the dentate gyrus was also included. The Schaffer collaterals were stimulated using bipolar electrodes placed in the CA3 region. Extracellular recordings were obtained in every experiment from the CA1 pyramidal cell layer. $A l v$, Alveus; $S c h$, Schaffer collaterals; $G C$, ganule cells; $M F$, mossy fibers; $P P$, perforant path; $B$, basket cell. the control of astrocyte swelling (Walz and Hertz, 1984; Kimelberg and Frangakis, 1985; Walz and Hinks, 1985; Walz, 1987; Walz and Mukerji, 1988). Physiological increases of external $\mathrm{K}^{+}$have been shown to cause $\mathrm{KCl}$ accumulation in cultured astrocytes through a furosemide-sensitive mechanism. The cellular mechanism is believed to be $\mathrm{Na}-\mathrm{K}-2 \mathrm{Cl}$ cotransport, which is accompanied by water movement into the cell and swelling. Accumulation of $\mathrm{Na}^{+}$is prevented by the $\mathrm{Na}-\mathrm{K}$ pump (Walz and Hinks, 1986). Activity-dependent swelling of astrocytes in the optic nerve also appears to involve $\mathrm{Na}-\mathrm{K}-2 \mathrm{Cl}$ cotransport because it is blocked by furosemide and low external $\mathrm{Cl}^{-}$(Ransom et al., 1985).

In this study, we have used imaging analysis techniques to examine changes in the intrinsic optical properties of hippocampal slices induced by synaptic transmission. We have also explored the influence of inhibiting the Na-K-2 $\mathrm{Cl}^{-}$cotransport exchanger with furosemide or low external $\mathrm{Cl}^{-}$(Geck et al., 1980; Geck and Heinz, 1986) to see if there are similarities between the results presented in previous studies of pharmacological manipulation of astrocyte swelling (e.g., Kimelberg and Frangakis, 1985; Ransom et al., 1985; Walz and Hinks, 1985) and the generation of optical signals in isolated slices.

Some of this work has been presented in abstract form (Hochman and MacVicar, 1990).

\section{Materials and Methods}

Tissue preparation. Hippocampal slices $(400 \mu \mathrm{m})$ were prepared from male Sprague-Dawley rats 3-6 weeks old. Isolated slices were maintained at room temperature $\left(21-23^{\circ} \mathrm{C}\right)$ in oxygenated $\left(95 \% \mathrm{O}_{2}, 5 \% \mathrm{CO}_{2}\right)$ artificial cerebral spinal fluid (aCSF) of the following composition: $\mathrm{NaCl}$, $126 \mathrm{~mm} ; \mathrm{KCl}, 5 \mathrm{~mm} ; \mathrm{MgCl}_{2}, 1.4 \mathrm{~mm} ; \mathrm{CaCl}_{2}, 2 \mathrm{~mm} ; \mathrm{NaHCO}_{3}, 26 \mathrm{~mm}$; glucose, $10 \mathrm{~mm}$. The low- $\mathrm{Cl}^{-}$solution contained the same constituents as the control aCSF except that $\mathrm{NaCl}$ was reduced to $6 \mathrm{~mm}$ and 120
mM Na-gluconate was added to maintain osmolarity. Therefore, $\mathrm{Cl}$ was reduced from 136.4 to $16.4 \mathrm{~mm}$. The $\mathrm{Ca}^{2+}$-free solution contained no added $\mathrm{CaCl}_{2}$, but EGTA ( $2 \mathrm{~mm}$ ) was added. The bicarbonate-free solution contained HEPES $(10 \mathrm{~mm})$ instead of $\mathrm{NaHCO}_{3}$ to maintain $\mathrm{pH}$, and the solution was aerated with $100 \% \mathrm{O}_{2}$. Some bicarbonate may be present in this solution because of the metabolic production of $\mathrm{CO}_{2}$ by the tissue at physiological pH (Roos and Boron, 1981).

Slices were individually transferred to a recording and imaging chamber (MacVicar, 1984) in which they were submerged in rapidly flowing $\operatorname{aCSF}\left(1.2 \mathrm{ml} / \mathrm{min} ; 21^{\circ} \mathrm{C}\right.$ or $\left.33^{\circ} \mathrm{C}\right)$. Slices were viewed from below through a $10 \times$ objective (neofluar or ultrafluar, Zeiss), and only a coverslip was between the slice and the objective. Electrodes were introduced from the top, and the slice was transilluminated. For extracellular recordings, microelectrodes $(\mathrm{NaCl}, 2 \mathrm{M} ; 10 \mathrm{M} \Omega)$ were placed in the CAl pyramidal layer (Fig. 1). Schaffer collaterals were stimulated by bipolar insulated silver stimulating electrodes in the CA3 region. In all experiments, the viability of the slice was tested and monitored throughout the experiment by observing the extracellularly evoked field potential.

Imaging analysis techniques. During the experiments, slices were transilluminated using a tungsten lamp that was powered by a voltageregulated power supply (Zeiss) that was stabilized so that voltage fluctuations were $<0.1 \%$. In some experiments, optical filters (20-nm bandwidth) were placed in the light path between the objective and the video camera. Video images were obtained using a CCD camera (Dage-MTI). The CCD camera was set for maximum gain with a high black level to filter out most of the extraneous background illumination and to increase the signal-to-noise ratio (discussed in Inoue, 1986). In most experiments, the image was linearly intensified using an image intensifier (model KS-1381, Videoscope), which we empirically found further increased the gain. The increase noise introduced by the image intensifier was eliminated using averaging techniques and digital filtering techniques (see below). Images were averaged and digitized using an Imaging Technology 151 Imaging Analysis System. The imaging system was controlled by an AT-compatible computer (Zenith 386), and images were stored digitally on a worm optical drive (Corel 940). The goal of the experiments was to examine alterations in the intrinsic optical properties of slices. Therefore, the experiments entailed acquiring a series of averaged images. Each image was usually an average of 128 frames, though in some experiments as few as 32 images were averaged. The 


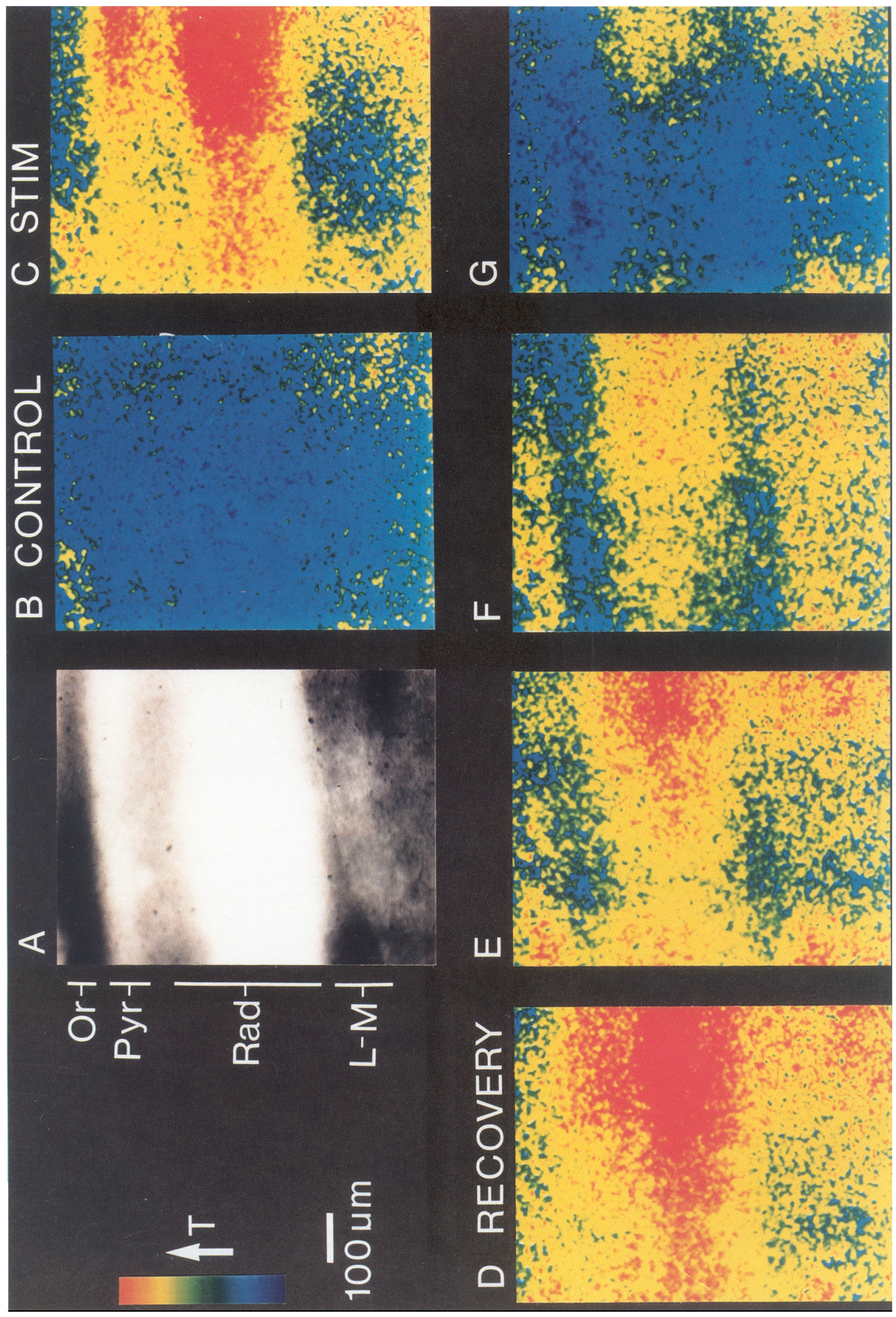



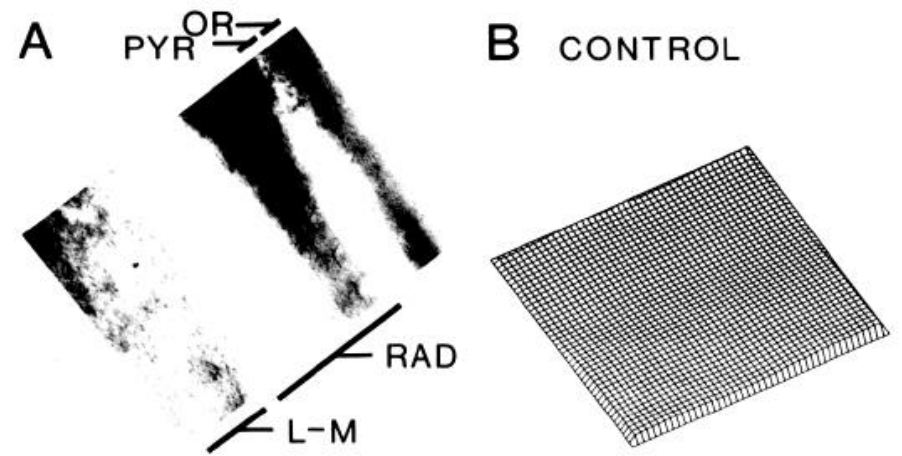

C $\operatorname{stim} 1 \mathrm{~Hz}$

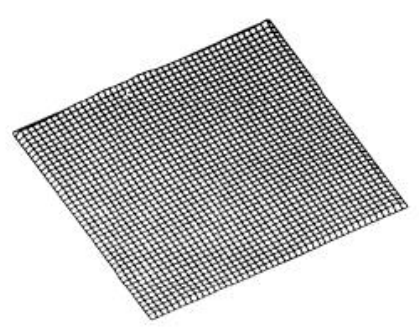

F $10 \mathrm{~Hz}$

E $5 \mathrm{~Hz}$

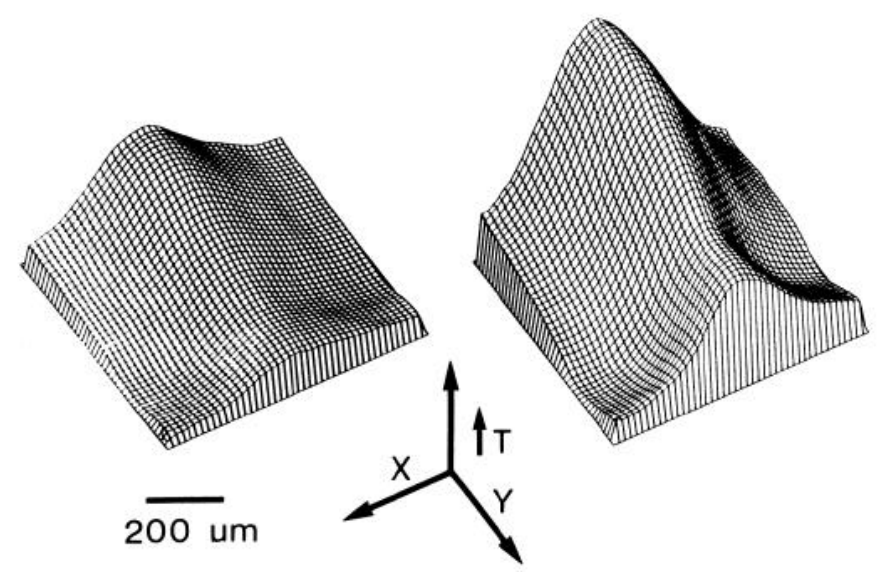

G $\quad 15 \mathrm{~Hz}$

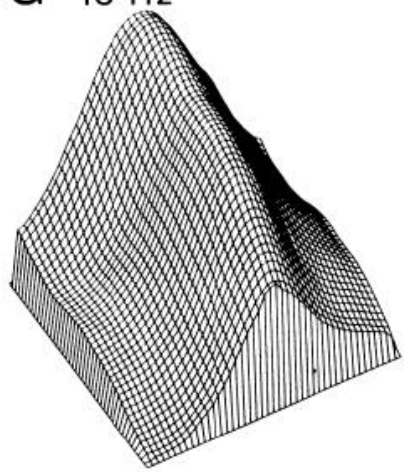

D $2 \mathrm{~Hz}$

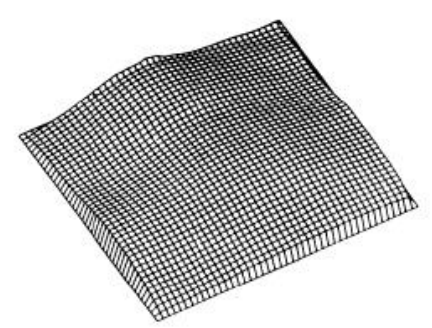

H $20 \mathrm{~Hz}$

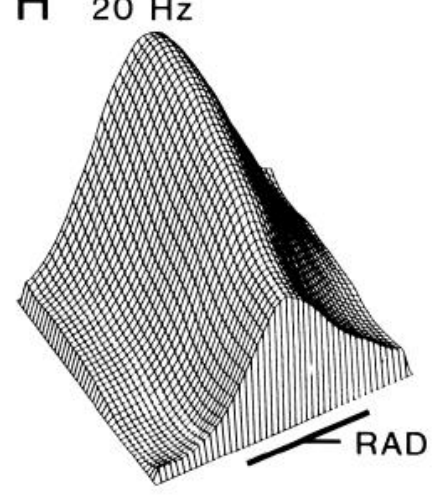

Figure 3. The magnitude of the change in the intrinsic optical signal increased with increasing stimulation frequencies. The control average image for this series is shown in $A$, and the control subtraction is shown in $B$. The intensities of the subtracted images are illustrated as 3-D surface plots where the z-axis (labeled $T$ ) represents the magnitude of the difference and therefore increased transmittance. The length of the $\mathrm{z}$-axis bar represents $\Delta T / T$ of $0.7 \%$. The peak response to stimulation at different frequencies, from 1 to $20 \mathrm{~Hz}$, is illustrated in $C-H$. In each frequency series, the slice was stimulated for $4 \mathrm{sec}$ at the indicated frequency and was observed to recover before the next series was attempted. At $1-\mathrm{Hz}$ stimulation, there was no detectable change, but at $2-\mathrm{Hz}$ stimulation there was a small increase. The increase in transmittance was of greater magnitude during 5and $10-\mathrm{Hz}$ stimulation and reached a maximum with $15-20-\mathrm{Hz}$ stimulation. The radiatum ( $\mathrm{Rad})$ was the area of maximal increase.

average images were obtained at video-frame rates $(30 \mathrm{~Hz})$. Therefore, it took approximately $4 \mathrm{sec}$ to acquire an average of 128 frames and 1 sec for 32 frames. The first image was used as a control, and it was subtracted from all subsequent images (total of 7 or 15 images). Therefore, the differences between the subtracted images showed the areas in which light transmission through the slice had changed. In all of the experiments in which pharmacological manipulations were attempted, we tested the stability of the intrinsic signals with three separate stimulus trials with recovery periods to ensure that the control responses were consistent.

The activity-dependent changes in light transmittance, $\Delta T / T$, were typically less than $2 \%$, whereas the noise, which was analyzed in every experiment by subtracting control images, was usually between $0.1 \%$ and $0.3 \%$. Signal-to-noise ratios ranged from 3 to 5 . The contrast of the subtracted images was enhanced by applying a linear transformation stretch to the image histogram (Inoue, 1986) to take advantage of the complete dynamic range of the 8-bit video system. The figures in this paper illustrate relative differences in light transmittance. The linearity of these relative differences was preserved throughout the analysis. Spike noise was removed from the images by neighborhood averaging and, in some cases, by additional median filtering. The subtracted frames were either displayed with the intensity differences color coded to make relative differences more obvious or with plots of surface maps of the pixel intensities. To make the surface map, a 100-to-1 data compression was accomplished by sampling the pixel value at every 10 th row and 10 th column of the analyzed image and converting the pixel values into an ASCII file. It was not necessary to use the total 256,000 pixels of an image because most of this data was redundant for the surface interpolation routine. We found empirically that the spatial resolution of the analyzed image was adequately preserved after this data compression. Commercially available software (sURFER, Golden Software) was used to generate the surface plot from this file.

Figure 2. Schaffer collateral stimulation induced an increase in light transmittance through the stratum radiatum. A series of seven images, of which each image represented an average of 128 video frames, were obtained $5 \mathrm{sec}$ apart. One average image is illustrated in $A$, and the layers within CAl can be observed. In this and all subsequent figures, the layers are labelled as $\operatorname{Or}$ (oriens), Pyr (pyramidale), $\operatorname{Rad}$ (radiatum), and $L$ - $M$ (lacunosum-moleculare). $B-G$ represent the subtraction of image 1 from images $2-7$, respectively. The magnitude of the differences, representing increased transmittance, are illustrated using a linear color-coded method in which the blue end of the spectrum depicts low-intensity transmittance differences $(\leq 0.3 \%$, background noise level) and red depicts high-intensity differences $(2 \%)$. The Schaffer collaterals were stimulated at $10 \mathrm{~Hz}$ for $4 \mathrm{sec}$ just before and during $C$. The radiatum where the Schaffer collaterals make excitatory synapses on CA1 dendrites exhibited the largest increase in light transmittance. Immediately following the cessation of stimulation $(D)$, the transmittance difference was still maximal. However, over the next $20 \sec (E-G)$, the light transmittance recovered to control levels. 


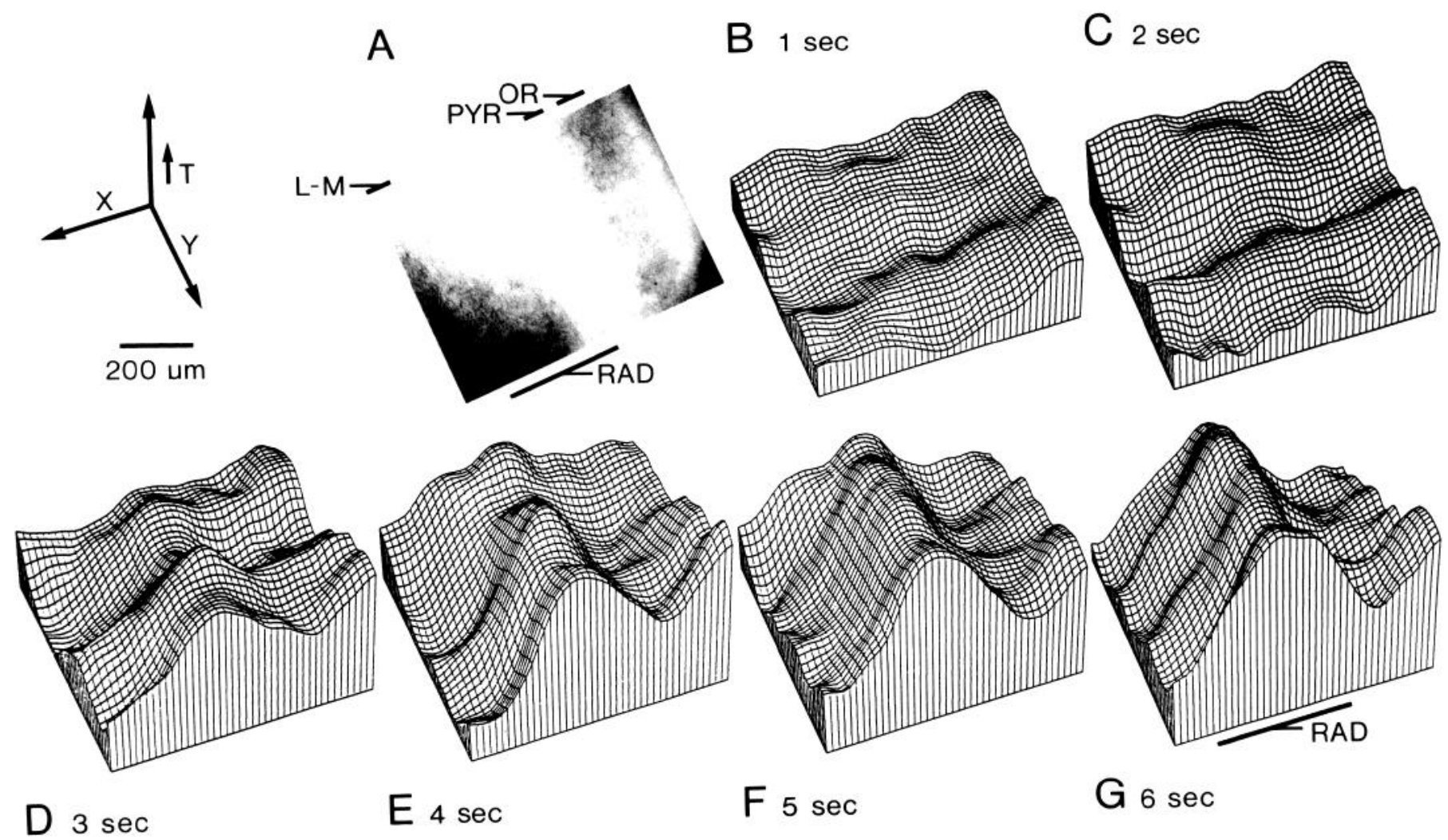

Figure 4. Time course of the onset of the intrinsic optical change. The averaged control image is shown in $A$, and the progressive increase in light transmittance is shown in $B-G$ at 1 -sec intervals. To determine the onset of the stimulus-evoked increase in light transmittance, averages were obtained of 32 video frames every second during a $10-\mathrm{Hz}$ stimulus train. At $2 \mathrm{sec}$ there was a barely detectable increase, whereas at $3 \mathrm{sec}$ the increase in light transmittance in the radiatum was obvious. At $6 \mathrm{sec}$ the pattern of light transmittance was well established. The length of the $z$-axis bar represents $\Delta T / T$ of $0.4 \%$.

\section{Results}

In all hippocampal slices ( $n=43$ from 20 animals) in which a synaptically evoked field potential could be recorded, stimulation of synaptic inputs at $10 \mathrm{~Hz}$ evoked an increase in light transmittance of the tissue. An example is illustrated in Figure 2 . The stimulating electrodes were positioned in the CA3 region out of the field of view (see Fig. 1). When the synaptic inputs to CAl were stimulated and field potentials were evoked, there was an increase in light transmission through the slice, particularly in the dendritic layer, where the Schaffer collateral inputs are localized. The increase in light transmittance gradually recovered to control levels when stimulation was stopped. The pyramidal cell body layer was always the area in the CAl region that exhibited the smallest amplitude increase.

The stimulation frequency was varied to see how the optical signal would be affected, and the results are illustrated in Figure 3 . In each series, the synaptic inputs were stimulated at the indicated frequency for $5 \mathrm{sec}$. For the last $4 \mathrm{sec}$ of the stimulation period, an averaged image of 128 video frames was acquired. The slice was then allowed to recover before the next series was attempted. The optical signal was monitored during recovery and always recovered to control levels after stimulation was stopped. At $2 \mathrm{~Hz}$, there was a slight change in light transmittance, which was much more obvious at $5 \mathrm{~Hz}$ and reached a peak at $15-20 \mathrm{~Hz}$. During most of the subsequent experiments, the slices were stimulated at $10 \mathrm{~Hz}$, which consistently gave a large signal. The experiments in this study required evoking several stimulus trains, and we chose the lower stimulus frequency of $10 \mathrm{~Hz}$ rather than $20 \mathrm{~Hz}$ to maintain slice viability for the longest time.

The time course of the onset of the optical signal during 10$\mathrm{Hz}$ stimulation and the recovery are illustrated in Figures 4 and 5 , respectively. To examine the onset of the intrinsic signal change, the slice was stimulated at $10 \mathrm{~Hz}$, and a series of seven averages of 32 frames (each therefore represents $1 \mathrm{sec}$ of acquisition) was obtained. An increase in light transmittance was first detectable at 2-3 sec of stimulation or after 20-30 stimuli (Fig. 4). By $6 \mathrm{sec}$, the intrinsic signal pattern was well established. To examine recovery, the slice was stimulated at $10 \mathrm{~Hz}$, and a series of averages of 128 frames (acquired over $4 \mathrm{sec}$ ) was obtained with a 1 -sec interval between each average image (Fig. 5). An increase in light transmittance was observed in the radiatum during $10-\mathrm{Hz}$ stimulation. When stimulation was stopped, light transmittance recovered to control levels within $20 \mathrm{sec}$.

Slices were then observed at different wavelengths every 50 $\mathrm{nm}$ from 450 to $800 \mathrm{~nm}$ using interference filters (20-nm bandpass) to examine the wavelength dependency of the intrinsic optical signal. As previously reported (MacVicar, 1984), hippocampal slices were much more transparent with increased contrast at longer wavelengths $(650-800 \mathrm{~nm})$. In the near infrared, cellular lamellae were much more apparent. The change in the intrinsic optical signal from synaptic stimulation was also much more pronounced with longer wavelengths. The largest amplitude signal was observed at $650 \mathrm{~nm}$, but the signal was 


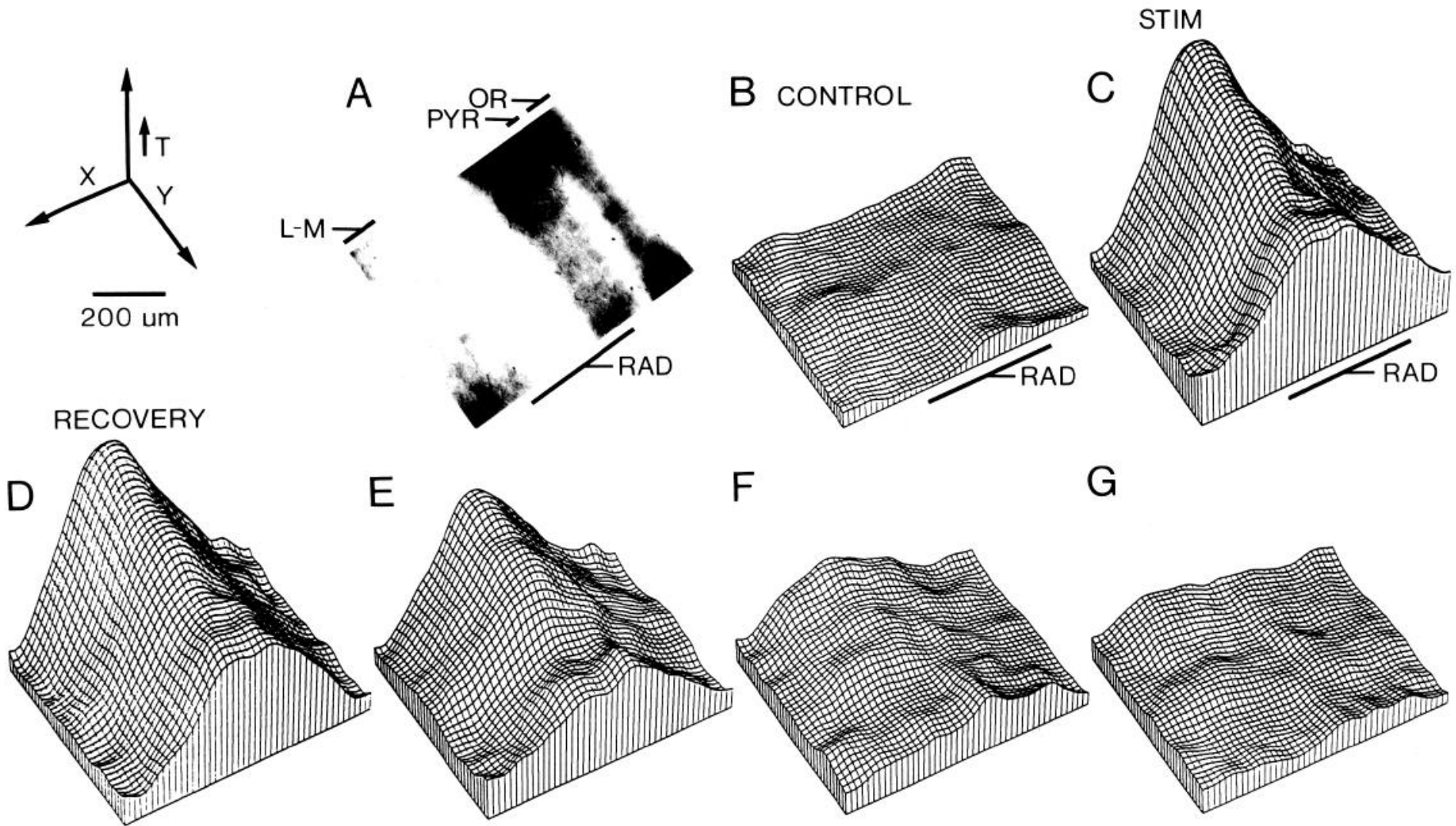

Figure 5. Time course of the recovery of increased light transmittance after the cessation of stimulation. Averages of 128 video frames were obtained during $10-\mathrm{Hz}$ stimulation of CA1. Each averaged image was acquired over a 4-sec time period with 1-sec intervals between averages. The control average is shown in $A$, and the control subtraction in $B$. Stimulation of the Schaffer collaterals $(C)$ caused an increase in light transmittance in the radiatum, as shown before. After cessation of the stimulation, light transmittance recovered to control levels $(D-G)$ within 20 sec. The length of the $z$-axis bar represents $\Delta T / T$ of $1.2 \%$.

not significantly greater than that recorded at $800 \mathrm{~nm}$ (Fig. 6; $n=3$ ). Therefore, the stimulus-evoked changes in the light transmittance in slices were observed over a wide range of wavelengths, which is characteristic of intrinsic optical signals. Typ- ically, we used $800-\mathrm{nm}$ or white light to observe slices because the structure and cellular layers of slices were much more apparent.

The next series of experiments was undertaken to determine

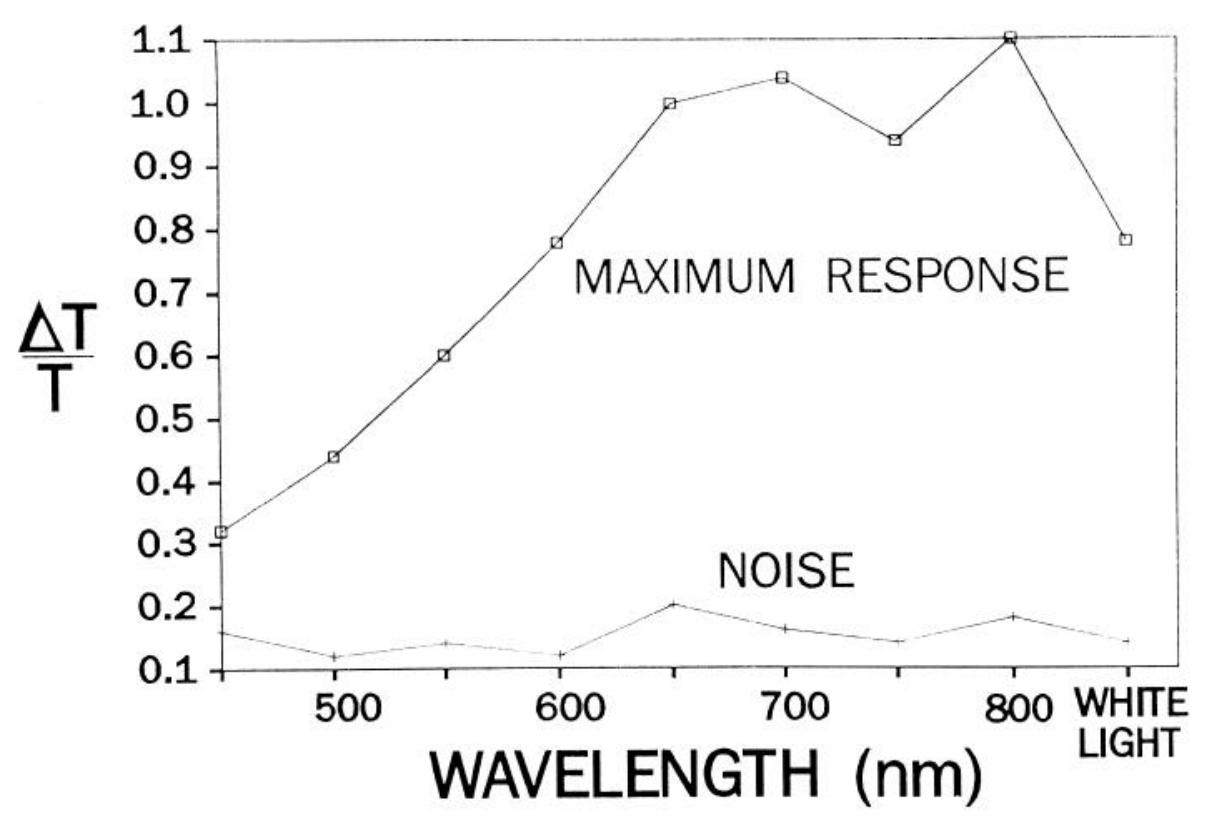

Figure 6. Wavelength dependency of the intrinsic optical changes. Following an experimental protocol similar to that illustrated in Figure 5, the Schaffer collaterals were stimulated at $10 \mathrm{~Hz}$. The changes in light transmittance were observed at different wavelengths every $50 \mathrm{~nm}$ from 450 to $800 \mathrm{~nm}$ and compared to the response observed with white light. Light illumination was changed to compensate for the increased transmittance at longer wavelengths so that baseline transmittance was equal at all wavelengths. The results from one experiment on one slice are illustrated, but consistent results were obtained from two other slices. After each stimulation and observation at the specified wavelength, the optical signal was always observed to recover to control levels. The peak response at each wavelength is plotted as $\Delta T / T \%$. Because $T$ was kept constant by changing illumination, this allows a comparison of $\Delta T$ with respect to wavelength. The noise level (measured in unstimulated controls) was fairly constant, but $\Delta T$ increased and stayed constant at wavelengths $>600 \mathrm{~nm}$. 


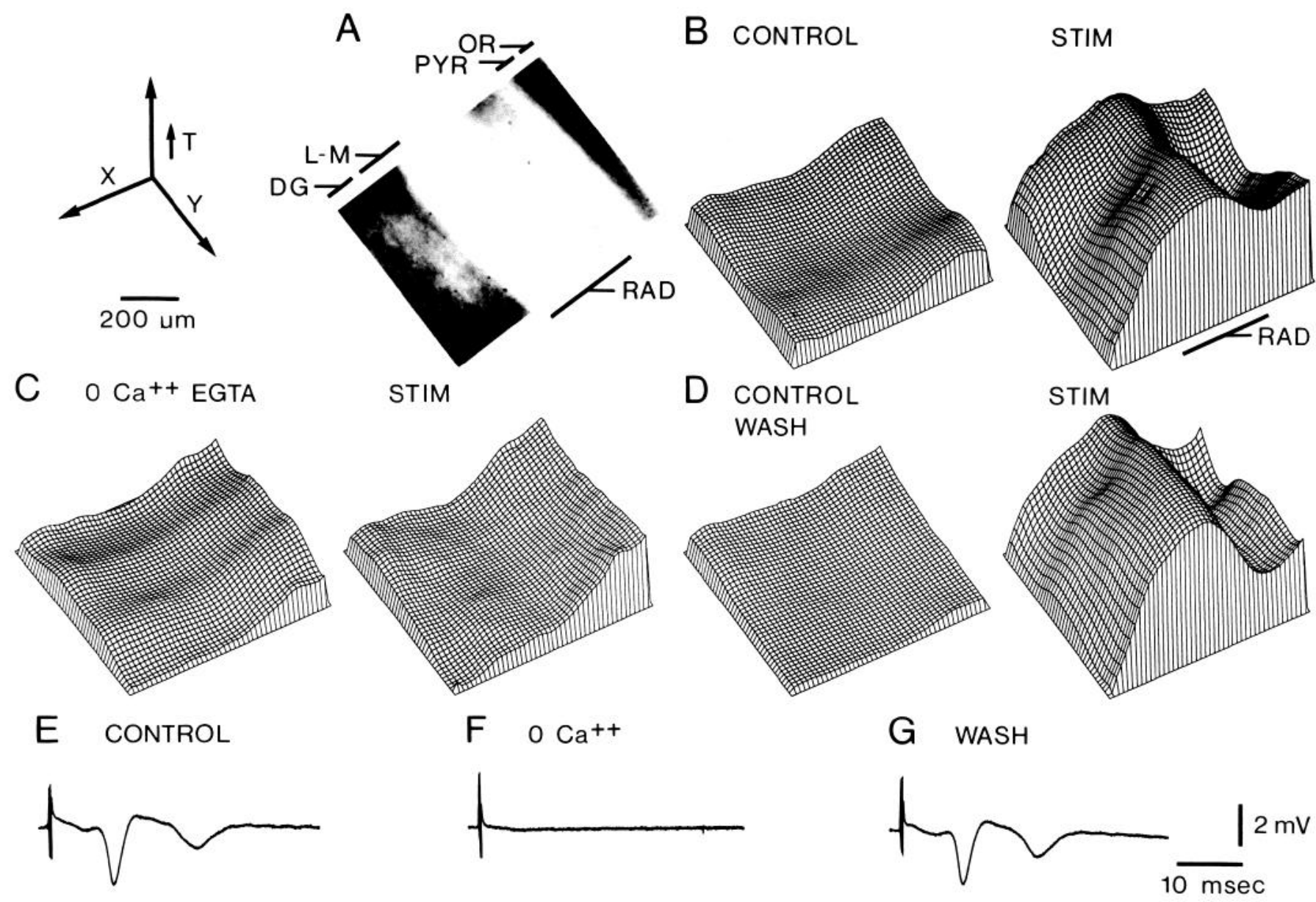

Figure 7. Blocking synaptic transmission with $0-\mathrm{Ca}^{2+}$ EGTA solution reversibly blocked the intrinsic optical changes. The averaged control image is shown in $A$, and the subtracted images are shown in $B-D$. The traces shown in $E-G$ represent extracellular recordings that were obtained from the pyramidal cell layer. In control solution, stimulation of the Schaffer collaterals at $10 \mathrm{~Hz}$ caused an increase in light transmittance in the radiatum. Extracellular stimulation evoked a population spike in CA1 $(E)$. In 0-Ca ${ }^{2+}$ EGTA solution, synaptic transmission was blocked $(F)$, and $10-\mathrm{Hz}$ stimulation did not cause any changes in light transmittance $(C)$. When control solution was reperfused with $\mathrm{Ca}^{2+}$, synaptic transmission recovered $(G)$, and $10-\mathrm{Hz}$ stimulation again caused an increase in light transmittance $(D)$. The length of the $z$-axis bar represents $\Delta T / T$ of $0.9 \%$.

the cellular basis for the changes in the intrinsic optical properties. We first investigated the role of synaptic transmission itself in generating the signals. Perfusing the slice with $\mathrm{Ca}^{2+}$-free EGTA solution blocked synaptic transmission in the CA1 region. When synaptic transmission was blocked (as indicated by extracellular recordings), the intrinsic signal was also blocked ( $n=7$; Fig. 7). The intrinsic signals were observed again after synaptic transmission recovered when control aCSF with $\mathrm{Ca}^{2+}$ was reperfused. This result indicated that either postsynaptic activation or the release of neurotransmitter itself was responsible for generating the signal. The next experiment was designed to differentiate between these two possibilities. Kynurenic acid, a wide-spectrum excitatory amino acid antagonist (Stone and Connick, 1985), was used to block synaptic transmission without blocking the release of transmitter. When synaptic transmission was shown to be blocked by kynurenic acid ( $1.5 \mathrm{~mm})$, the changes in the intrinsic optical signals were also blocked ( $n$ $=7$; Fig. 8). The intrinsic signals were again observed when synaptic transmission recovered upon washout of the kynurenic acid. Therefore, the changes in light transmittance result from postsynaptic activation and not just from exocytosis of neurotransmitters or presynaptic fiber activation.
It is possible that the increase in light transmittance is due to cellular swelling (Lipton, 1973) that is induced by postsynaptic activation. Although it is difficult to differentiate between neuronal or astrocyte swelling, astrocytes have been shown to be the most susceptible to swelling under these conditions. Astrocyte swelling from raised external $\mathrm{K}^{+}$during activity could be responsible for the changes in the extracellular space observed during neuronal activity (Ransom et al., 1985). Astrocyte swelling in tissue culture has also been shown to depend upon a furosemide-sensitive anion transporter, the $\mathrm{Na}-\mathrm{K}-2 \mathrm{Cl}$ cotransporter (Walz and Hinks, 1985). We therefore investigated the furosemide sensitivity of the intrinsic optical signals to see if this might provide some indirect evidence for the involvement of astrocytes. Perfusion of furosemide ( $5 \mathrm{mM}$ ) reversibly blocked the intrinsic optical signals ( $n=5$; Fig. 9) but did not block synaptic transmission. In fact, multiple population spikes were observed in furosemide. If a furosemide-sensitive $\mathrm{Cl}^{-}$transporter is involved, then reducing extracellular $\mathrm{Cl}^{-}$should also block the intrinsic signals. Reducing extracellular $\mathrm{Cl}^{-}$by equimolar replacement with gluconate also reversibly blocked the intrinsic signals ( $n=7$; Fig. 10). Synaptic transmission was not decreased by the low- $\mathrm{Cl}^{-}$solution. Reducing extracellular bi- 


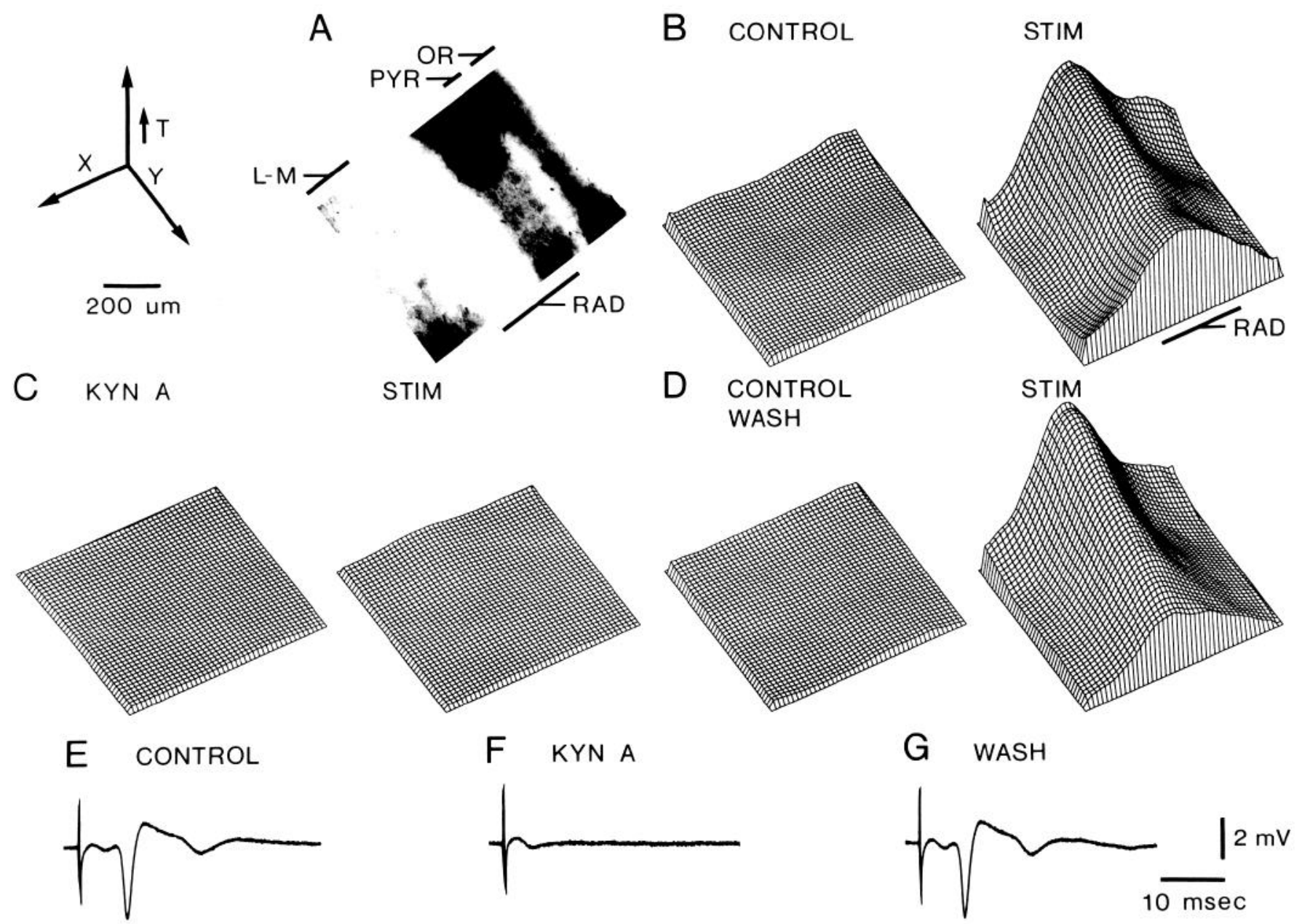

Figure 8. Blocking synaptic transmission with a postsynaptic receptor antagonist, kynurenic acid, reversibly blocked the intrinsic optical changes. The average control image is shown in $A$, and the subtracted images are illustrated in $B-D$. Extracellular recordings in the pyramidal cell layer of CA1 from Schaffer collateral stimulation are shown in $E-G$. Stimulation of the Schaffer collaterals at $10 \mathrm{~Hz}$ caused an increase in light transmittance in the radiatum. After the perfusion of kynurenic acid $(K Y N A ; 1.5 \mathrm{~mm})$, synaptic transmission was blocked $(F)$, and $10-\mathrm{Hz}$ stimulation of the Schaffer collaterals did not cause any change in light transmittance in the slice $(C)$. When control solution was reperfused, synaptic transmission recovered $(G)$, and $10-\mathrm{Hz}$ stimulation again caused an increase in light transmittance in the radiatum $(D)$. The length of the $z$-axis bar represents $\Delta T / T$ of $0.9 \%$.

carbonate by exchanging the control perfusate with a HEPESbuffered solution that was aerated with $100 \% \mathrm{O}_{2}$ had no effect on the intrinsic optical signals $(n=4$; Fig. 10). Therefore, a furosemide-sensitive $\mathrm{Cl}^{-}$bicarbonate cotransport does not appear to be involved.

\section{Discussion}

The results presented here indicate that synaptic activity causes an increase in light transmittance in the dendritic area of CA1. The pyramidal cell body layer does not exhibit the increase in light transmittance to the same extent. This increase summates with repetitive stimulation and slowly recovers when the stimulation is stopped. Because this increase was observed in the isolated hippocampal slice, it is obvious that changes in blood flow do not contribute to this signal generation. A component of the intrinsic signal observed in vivo may be due to changes in blood flow (Lieke et al., 1989).

The pharmacological manipulations provide some evidence for the cellular mechanisms that may underlie the changes in the intrinsic optical properties of the tissue. The signal is not generated by presynaptic fiber volleys or by exocytosis of neu- rotransmitter. When synaptic transmission was blocked by $\mathrm{Ca}^{2+}$ free EGTA perfusate, the change in the intrinsic optical signal was blocked. This solution would not, however, block action potentials in the presynaptic fibers. When synaptic transmission was blocked by a receptor antagonist, kynurenic acid, the change in the intrinsic optical signal was also blocked. Exocytosis of neurotransmitters would still occur in this solution. Therefore, postsynaptic activation is necessary for the change in the intrinsic signal to be generated. Presynaptic fiber volleys and release of transmitter are not sufficient to induce these optical changes. In the mammalian neurohypophysis, it is thought that neurosecretion alone is sufficient to generate large changes in light scattering (Salzberg et al., 1985). This is apparently not the case in the hippocampus. In the posterior pituitary, peptides are released from dense-core vesicles, whereas glutamate is most likely released from smaller synaptic vesicle at the Schaffer collateral-CA1 synapse. The differences in the types of vesicles could perhaps explain the differences between the generation of intrinsic signals in the two systems. It is also possible that the changes in intrinsic signals in the pituitary are caused by morphological changes in pituicytes induced by the release of neu- 


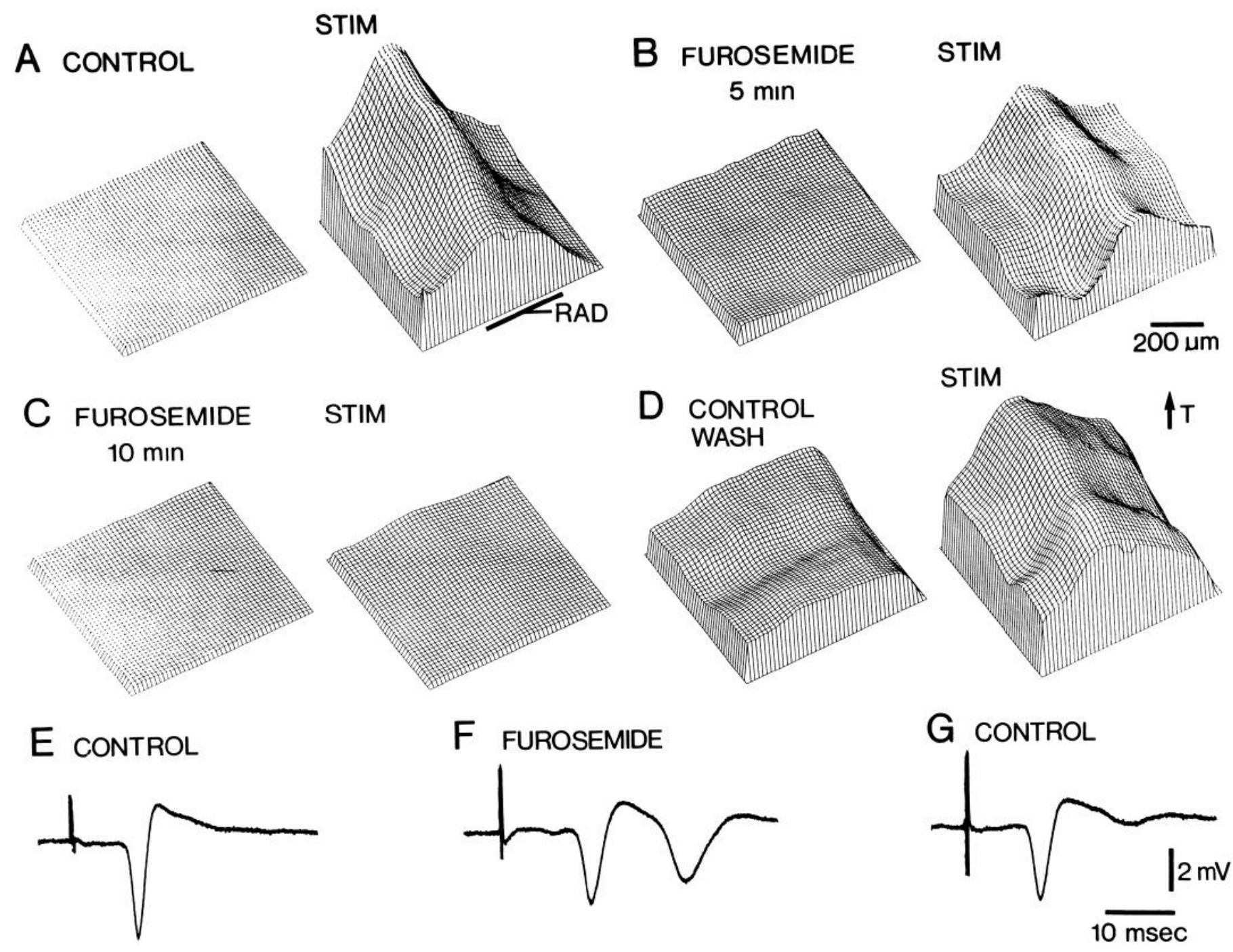

Figure 9. Furosemide, an anion transport inhibitor, blocked the intrinsic optical signal. In control solution, extracellular stimulation of the Schaffer collaterals evoked a population spike in the CAl pyramidal cell layer $(E)$ and caused an increase in light transmittance through the dendritic radiatum area $(A)$. Perfusion of furosemide $(5 \mathrm{~mm})$, an anion transport inhibitor, progressively decreased the stimulation-evoked change in light transmittance. At $5 \mathrm{~min}$ the change was decreased $(B)$, and at $10 \mathrm{~min}(C)$ there was no detectable change in light transmittance through the radiatum. However, synaptic transmission was not blocked after $10 \mathrm{~min}$ of perfusion of furosemide $(F)$. A second population spike was observed that may result from furosemide's actions on inhibition. After furosemide was washed from the slice and control solution was perfused for approximately $60 \mathrm{~min}$, Schaffer collateral stimulation again caused an increase in light transmittance $(D)$, and only one population spike was evoked with extracellular stimulation $(G)$. The length of the $z$-axis bar represents $\Delta T / T$ of $0.2 \%$.

rotransmitters or hormones (Hatton, 1988; Bicknell et al., 1989). It should be noted that it is difficult to compare directly the intrinsic optical signals that we have recorded using imaging techniques with previous reports using photodiodes (e.g., Grinvald et al., 1982; Salzberg et al., 1985). The photodiode measurements were all AC coupled (coupling time constants of 100 msec to $3 \mathrm{sec}$ ), whereas the imaging measurements of light intensity could be considered DC coupled. The time to obtain one image (4 sec to average 128 frames) also means that prolonged steady changes in light intensity that are filtered in photodiode experiments will predominately influence imaging experiments.

From our experiments with furosemide and $\mathrm{Cl}^{-}$replacement, we concluded that the change in the intrinsic optical properties was dependent upon $\mathrm{a} \mathrm{Cl}^{-}$transport mechanism. Furosemide, which inhibits anion transport (Geck et al., 1980; Geck and Heinz, 1986), and reduced extracellular $\mathrm{Cl}^{-}$blocked the stim- ulus-evoked changes in light transmittance. The changes in light transmittance were blocked even though extracellular stimulation evoked population spikes, indicating that the pyramidal cells were still synaptically activated. Reduction of extracellular bicarbonate did not, however, alter the intrinsic signal. Furosemide inhibits both a $\mathrm{Cl}^{-}$bicarbonate exchanger (Bourke et al., 1983 ) and $\mathrm{Na}-\mathrm{K}-2 \mathrm{Cl}$ cotransport. Although metabolic production of $\mathrm{CO}_{2}$ may create some bicarbonate in the tissue at physiological pH (Roos and Boron, 1981), our observations that nominally bicarbonate-free perfusate had no effect indicate that $\mathrm{Na}-\mathrm{K}-2 \mathrm{Cl}$ cotransport is most likely the exchanger involved.

There are similarities between the pharmacological sensitivity of the stimulus-evoked changes in light transmittance reported here and the activity-dependent shrinkage of the extracellular space in the optic nerve reported by Ransom et al. (1985). The time course of the two are also quite similar. Stimulation of the 
A CONTROL

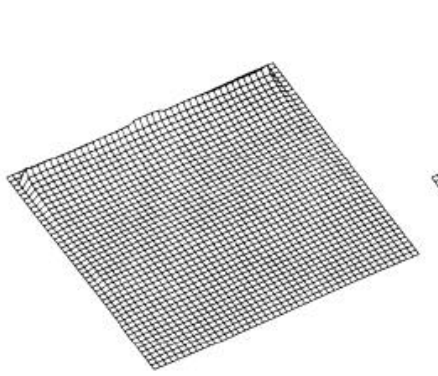

C GLUCONATE
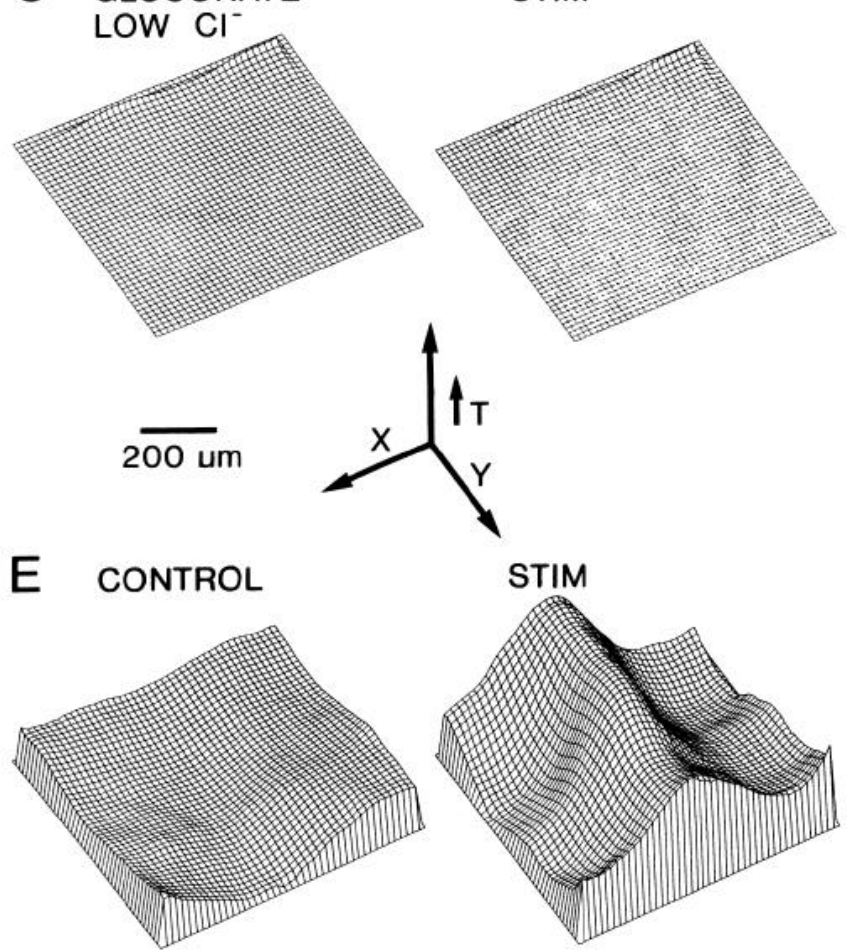

\section{STIM}

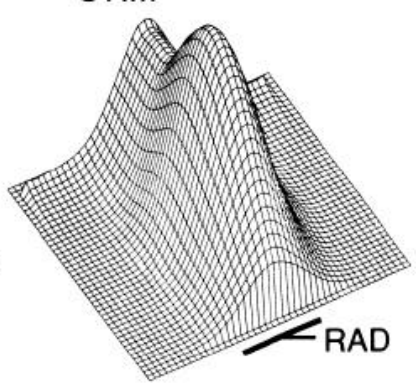

STIM
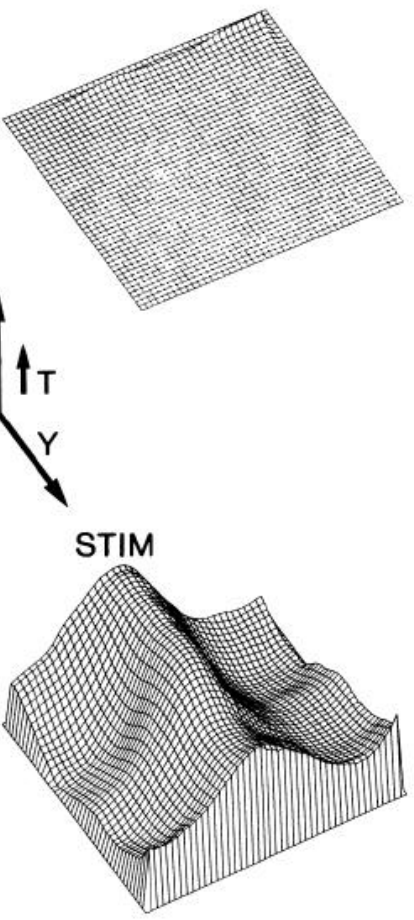

B CONTROL

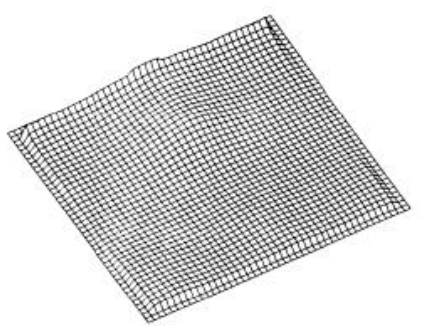

D CONTROL WASH
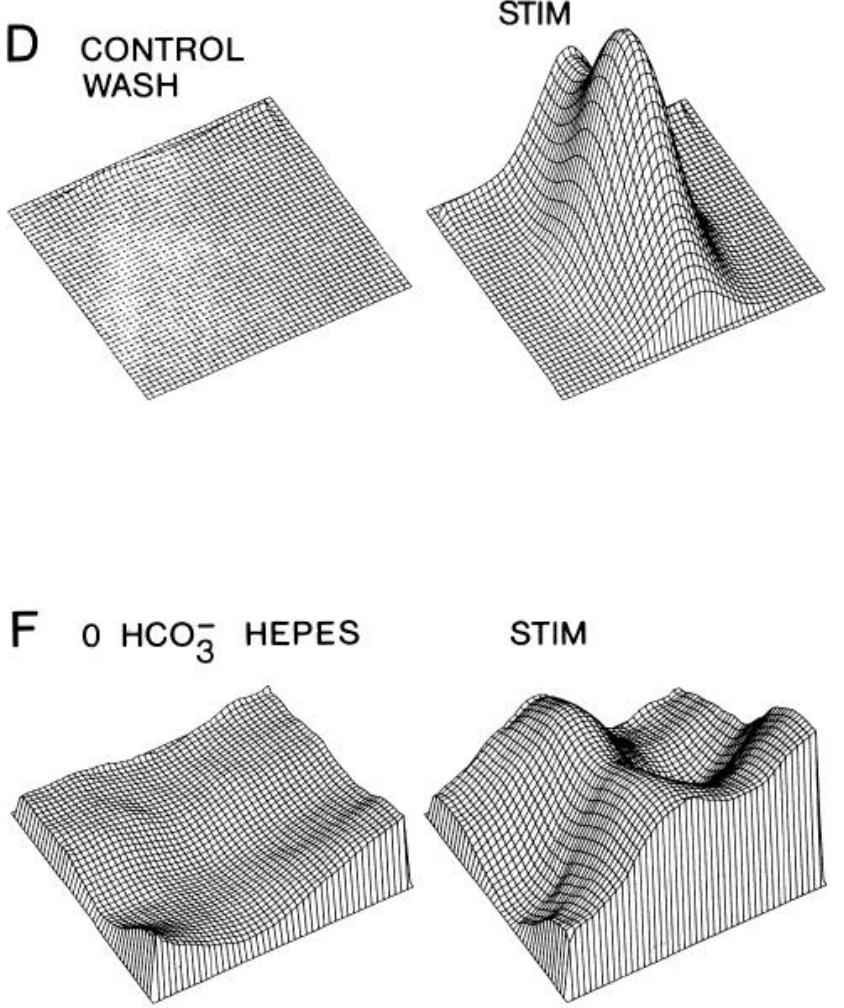

Figure 10. Reducing extracellular chloride by replacement with gluconate blocked the intrinsic optical signal, but the signal was still recorded in bicarbonate-free solution. In control solution, $10-\mathrm{Hz}$ stimulation of the Schaffer collaterals caused an increase in light transmittance in the dendritic radiatum area of $\mathrm{CA} 1(A)$. The response in control solution was stable and exhibited the same increase in light transmittance with a second trial of $10-\mathrm{Hz}$ stimulation $(B)$. When extracellular $\mathrm{Cl}^{-}$was reduced to $8 \mathrm{~mm}$ by equimolar replacement with gluconate, 10- $\mathrm{Hz}$ stimulation of the Schaffer collaterals, which still evoked population spikes, did not cause any change in light transmittance in the slice $(C)$. When control solution was reperfused, $10-\mathrm{Hz}$ stimulation of the Schaffer collaterals again caused an increase in light transmittance in the radiatum. In another experiment, we examined the action of reducing extracellular bicarbonate. In control solution $(E), 10-\mathrm{Hz}$ stimulation of the Schaffer collaterals caused an increase in light transmittance through the radiatum. When the slice was perfused with a bicarbonate-free solution that was aerated with $100 \% \mathrm{O}_{2}$ and $\mathrm{pH}$ buffered with HEPES, $10-\mathrm{Hz}$ stimulation of the Schaffer collaterals still caused an increase in light transmittance in the radiatum $(F)$. The length of the $z$-axis bar represents $\Delta T / T$ of $0.7 \%$ in $A-D$ and $1.2 \%$ in $E$ and $F$.

optic nerve was found to decrease the extracellular space, and this decrease was blocked by furosemide and by $\mathrm{Cl}^{-}$-free solution. Ransom et al. (1985) also examined the onset of activitydependent shrinkage of the extracellular space during postnatal development and found that it was correlated with the differentiation of astrocytes. It is possible that activity-dependent shrinkage of the extracellular space occurs because of swelling of astrocytes. Anatomical studies have suggested that raised external $\mathrm{K}^{+}$causes swelling primarily in astrocytes in vivo (reviewed in Hertz, 1981; Kimelberg and Ransom, 1986). In primary tissue culture, high external $\mathrm{K}^{+}$causes swelling of astro- cytes due to $\mathrm{KCl}$ uptake and water movements (Walz and Hinks, 1985). This can be blocked by furosemide (Bourke et al., 1983; Walz and Hertz, 1984; Kimelberg and Frangakis, 1985) and reduced external $\mathrm{Cl}^{-}$, indicating that astrocyte swelling depends upon Na-K-2Cl cotransport. The glial cells do not accumulate $\mathrm{Na}^{+}$because of a transmembrane $\mathrm{Na}^{+}$cycle (Walz and Hinks, 1986). Na-K-2Cl cotransport is also activated during swelling or regulatory volume increases in other cell types such as Ehrlich ascites tumor cells (Hoffmann et al., 1983).

Is it possible that swelling of astrocytes could underlie the increases in light transmittance that occur during synaptic trans- 
mission in CA1? A possible scenario is that extracellular $\mathrm{K}^{+}$ increases during synaptic transmission, which increases Na-K$2 \mathrm{Cl}$ cotransport into astrocytes, and that this leads to $\mathrm{KCl}$ and water accumulation. A direct action of glutamate on astrocytes is possible but unlikely because furosemide, which blocked the intrinsic signal, has not been shown to disrupt glutaminergic responses. Cellular swelling within the slice could very well lead to increased light transmittance. The increase in light transmittance that we observed during synaptic activity in slices may be analogous to the decreased reflectance in slices and the decreased light scattering in cell and organelle suspensions during osmotically induced volume increases (discussed in Cohen, 1973; Lipton, 1973). These optical changes during volume increase presumably result from the decrease in the refractive index difference between cytoplasm and extracellular solution (Cohen and Keynes, 1971; Lipton, 1973). Neuronal swelling could also occur due to synaptically activated currents, but this appears to be less likely. In both furosemide and reduced external $\mathrm{Cl}^{-}$, synaptic activity was still evoked, though the changes in light transmittance were blocked. Also, the pyramidal cell layer did not exhibit the same increase in light transmittance that was observed in the dendritic region. If neuronal swelling underlies the changes in light transmission, it might be expected that the cell bodies would swell as well as the dendrites. The change in light transmission also had a much slower time course than synaptic transmission. During repetitive synaptic stimulation at $10 \mathrm{~Hz}$, the first changes in light transmittance were detected after $3 \mathrm{sec}$, and the signal slowly recovered over $20 \mathrm{sec}$ after stimulation ceased. Therefore, the changes in the tissue occur much more slowly than synaptically activated currents and the associated ion flows. However, neuronal and dendritic swelling have been reported during seizure activity and spreading depression (Van Harreveld and Khattab, 1967; Van Harreveld, 1972). A component of the intrinsic optical signal that we have observed could be due to neuronal swelling. However, numerous studies, as discussed above, have indicated that astrocytes are most sensitive to cellular swelling.

A recent report has indicated that there are regional variations in the volume fraction of the extracellular space in the hippocampus (McBain et al., 1990). The extracellular volume fraction in the stratum pyramidal layer of CA 1 was the smallest as compared to C.A3 and the dentate gyrus, and it decreased during increased external $\mathrm{K}^{+}$. Synchronization of neuronal spiking in the hippocampus can be influenced by alterations in the extracellular volume (Andrew et al., 1989; Traynelis and Dingledine, 1989), presumably because of the resultant changes in extracellular resistance and ephaptic interactions between neurons (Taylor and Dudek, 1982). The spatial distribution within CAl of increased light transmittance reported in this study may reflect regional variations of cellular swelling and extracellular volume changes. We would therefore predict that there should be a laminar distribution of extracellular volume decreases and possibly extracellular resistance increases that mirrors the increased light transmittance. The largest decreases in extracellular volume should therefore be observed in the radiatum. This would also imply that ephaptic interactions would be enhanced preferentially in this area, as predicted by computer modeling (Traub et al., 1985). The analysis of the laminar distribution of activity-dependent extracellular volume changes within CA1 has not been accomplished to date to our knowledge.

The changes in light transmittance were observed when the slice was stimulated at $10 \mathrm{~Hz}$, which is the frequency of the naturally occurring $\theta$-rhythm $(4-10 \mathrm{~Hz})$ in the hippocampus (Bland, 1986). In the slice preparation, $\theta$-oscillations can be induced in the population of cells by perfusion of a muscarinic agonist (MacVicar and Tse, 1989). The alterations in the intrinsic optical properties of the tissue and the underlying physiological changes that these optical changes represent, whatever they are, could be occurring during this naturally developing rhythm.

Changes in the intrinsic optical properties of neuronal tissue should always be considered with optical imaging techniques using voltage- or ion-sensitive dyes. Intrinsic optical signals themselves may be useful for imaging patterns of activity in the CNS. Ocular dominance columns and orientation columns in cat and primate visual cortex have been imaged by observing changes in reflectance (Grinvald et al., 1986; Ts'o et al., 1990). The patterns were similar to those observed using voltage-sensitive dyes (Blasdel and Salama, 1986). However, it is not necessary to use physiologically invasive dyes to detect changes in the intrinsic optical properties of neuronal tissue. We have observed activity-dependent changes in the intrinsic optical properties of the human cortex and have used it to map the spread of seizure activity (MacVicar et al., 1990). The data presented in this study provide initial clues concerning the cellular basis for changes in intrinsic signals and are an important step in advancing the utility of this noninvasive technique.

\section{References}

Andrew RD, Fagan M, Ballyk BA, Rosen AS (1989) Seizure susceptibility and the osmotic state. Brain Res 498:175-180.

Bicknell RJ, Luckman SM, Inenaga K, Mason WT, Hatton GI (1989) $\beta$-Adrenergic and opioid receptors on pituicytes cultured from adult rat neurohypophysis: regulation of cell morphology. Brain Res Bull 22:379-388.

Bland BH (1986) The physiology and pharmacology of hippocampal formation theta rhythms. Prog Neurobiol 26:1-54.

Blasdel GG, Salama G (1986) Voltage-sensitive dyes reveal a modular organization in monkey striate cortex. Nature 321:579-585.

Bourke RS, Kimelberg HK, Daze M, Church G (1983) Swelling and ion uptake in cat cerebrocortical slices: control by neurotransmitters and ion transport mechanisms. Neurochem Res 8:5-24.

Cohen LB (1973) Changes in neuron structure during action potential propagation and synaptic transmission. Physiol Rev 53:373-418.

Cohen LB, Keynes RD (1971) Changes in light scattering associated with the action potential in crab nerves. J Physiol (Lond) 212:259275.

Dietzel I, Heinemann U, Hofmeier G, Lux HD (1980) Transient changes in the size of the extracellular space in the sensorimotor cortex of cats in relation to stimulus induced changes in potassium concentration. Exp Brain Res 40:432-439.

Dietzel I, Heinemann U, Hofmeier G, Lux HD (1982) Stimulusinduced changes in extracellular $\mathrm{Na}^{+}$and $\mathrm{Cl}^{-}$concentration in relation to changes in the size of the extracellular space. Exp Brain Res 46: $73-84$.

Geck P, Heinz E (1986) The Na-K-2Cl cotransport system. J Membr Biol 91:97-105.

Geck P, Pictrzyk C, Burckhardt BC, Pfeiffer B, Heinz E (1980) Electrically silent cotransport of $\mathrm{Na}^{+}, \mathrm{K}^{+}$and $\mathrm{Cl}^{-}$in Ehrlich cells. Biochim Biophys Acta 600:432-477.

Grinvald A, Manker A, Segal M (1982) Visualization of the spread of electrical activity in rat hippocampal slices by voltage-sensitive optical probes. J Physiol (Lond) 333:269-291.

Grinvald A, Lieke E, Frostig RD, Gilbert CD, Wiesel TN (1986) Functional architecture of cortex revealed by optical imaging of intrinsic signals. Nature 324:361-364.

Hatton GI (1988) Pituicytes, glia and control of terminal secretion. J Exp Biol 139:67-79.

Heinemann U, Dietzel I (1984) Extracellular potassium concentration in chronic alumina cream foci of cats. J Neurophysiol 52:421-434.

Heinemann U, Neuhaus S, Dietzel I (1983) Aspects of potassium 
regulation in normal and gliotic brain tissue. In: Current problems in epilepsy, I, Cerebral blood flow, metabolism and epilepsy (BaldyMoulinier M, Ingvar DH, Meldrum BS, eds), pp 271-277. London: Libbey.

Hertz L (1981) Features of astrocytic function apparently involved in the response of central nervous tissue to ischemia-hypoxia. J Cereb Blood Flow Metab 1:143-153.

Hill DK, Keynes RD (1949) Opacity changes in stimulated nerve. J Physiol (Lond) 108:278-281.

Hochman D, MacVicar BA (1990) Imaging of intrinsic optical signals in hippocampal slices during synaptic activation. Soc Neurosci Abstr 16:1096.

Hoffmann EK, Sjoholm C, Simonsen LO (1983) $\mathrm{Na}^{+}, \mathrm{Cl}^{-}$cotransport in Ehrlich ascites tumor cells activated during volume regulation (regulatory volume increasc). J Membr Biol 76:269-280.

Inoue S (1986) Video microscopy. New York: Plenum.

Kimelberg HK, Frangakis MV (1985) Furosemide- and bumetanidesensitive ion transport and volume control in primary astrocyte cultures from rat brain. Brain Res 61:125-134.

Kimelberg HK, Ransom BR (1986) Physiological and pathological aspects of astrocyte swelling. In: Astrocytes: cell biology and pathology of astrocytes, Vol 3 (FederoffS, Vernadakis A, eds), pp 129-166. New York: Academic.

Lieke EE, Frostig RD, Arieli A, Ts'o DY, Hildesheim R, Grinvald A (1989) Optical imaging of cortical activity: real-time imaging using extrinsic dye-signals and high resolution imaging based on slow intrinsic-signals. Annu Rev Physiol 51:543-559.

Lipton P (1973) Effects of membrane depolarization on light scattering by ccrcbral cortical slices. J Physiol (Lond) 231:365-383.

MacVicar BA (1984) Infrared video microscopy to visualize neurons in the in vitro brain slice preparation. J Neurosci Methods 12:133139.

MacVicar BA, Tse FWY (1989) Local neuronal circuitry underlying cholinergic rhythmical slow activity in $\mathrm{CA} 3$ area of rat hippocampal slices. J Physiol (Lond) 417:197-212.

MacVicar BA, Hochman D, LeBlanc FE, Watson TW (1990) Stimulation evoked changes in intrinsic optical signals in the human brain. Soc Neurosci Abstr 16:309.

McBain CJ, Traynelis SF, Dingledine R (1990) Regional variation of extracellular space in the hippocampus. Science 249:674-677.

Obaid AL, Flores R, Salzberg BM (1989) Calcium channels that are required for secretion from intact nerve terminals of vertebrates are sensitive to $w$-conotoxin and relatively insensitive to dihydropyridines. J Gen Physiol 93:715-729.

Orkand RK, Dietzel I, Coles JA (1984) Light-induced changes in extracellular volume in the retina of the drone, Apis mellifera. Neurosci Lett 45:273-278.
Ransom BR, Yamate CL, Connors BW (1985) Activity-dependent shrinkage of axtracellular space in rat optic nerve: a developmental study. J Neurosci 5:532-535.

Roos A, Boron WF (1981) Intracellular pH. Physiol Rev 221:296434.

Salzberg BM, Obaid AL, Gainer H (1985) Large and rapid changes in light scattering accompany secretion by nerve terminals in the mammalian neurohypophysis. J Gen Physiol 86:887-908.

Serve G, Endres W, Grafe P (1988) Continuous electrophysiological measurements of changes in cell volume of motoneurons in the isolated frog spinal cord. Pfluegers Arch 411:410-415.

Snow RW, Taylor CP, Dudek FE (1983) Electrophysiological and optical changes in slices of rat hippocampus during spreading depression. J Neurophysiol 50:561-572.

Stonc TW, Connick JH (1985) Quinolinic acid and other kynurenines in the central nervous system. Neuroscience 15:597-617.

Taylor CP, Dudek FE (1982) Synchronous neural afterdischarges in rat hippocampal slices without active chemical synapses. Science 218: 810-812.

Traub RD, Dudek FE, Snow RW, Knowles WD (1985) Computer simulations indicate that electrical field effects contribute to the shape of the epileptiform field potential. Neuroscience 15:947-958.

Traynelis SF, Dingledine R (1989) Role of extracellular space in hyperosmotic suppression of potassium-induced electrographic seizures. J Neurophysiol 61:927-934.

Ts'o DY, Frostig RD, Lieke EE, Grinvald A (1990) Functional organization of primate visual cortex revealed by high resolution optical imaging. Science 249:417-420.

Van Harrcveld A (1972) The extracellular space in the vertebratc central nervous system. In: The structure and function of nervous tissue (Bourne GH, ed), pp 449-511. New York: Academic.

Van Harreveld A, Khattab FI (1967) Changes in cortical extracellular space during spreading depression investigated with the electron microscope. J Neurophysiol 30:911-929.

Walz W (1987) Swelling and potassium uptake in cultures astrocytes. Can J Physiol Pharmacol 65:1051-1057.

Walz W, Hertz L (1984) Intense furosemide-sensitive potassium accumulation in astrocytes in the presence of pathologically high extracellular potassium levels. J Cereb Blood Flow Metab 4:301-304.

Walz W, Hinks EC (1985) Carrier-mediated $\mathrm{KCl}$ accumulation accompanied by water movements is involved in the control of physiological $\mathrm{K}^{+}$levels by astrocytes. Brain Res 343:44-51.

Walz W, Hinks EC (1986) A transmembrane sodium cycle in astrocytes. Brain Res 368:226-232.

Walz W, Mukerji S (1988) KCl movements during potassium-induced cytotoxic swelling of cultured astrocytes. Exp Neurol 99:17-29. 